\title{
Rescuing mitochondrial motility
}

mitofusin
agonists
corrected the
mitochondrial
dysmorphology
and
reversed the
mitochondrial
hypo-
polarization

Charcot-Marie-Tooth disease type $2 \mathrm{~A}(\mathrm{CMT} 2 \mathrm{~A})$ is caused by mutations in the gene encoding mitofusin 2 (MFN2) that suppress mitochondrial fusion and motility, and is characterized by severe progressive muscle weakness, motor deficits and peripheral neuropathy. There are currently no therapeutics that directly enhance mitochondrial fusion or trafficking, therefore the disease is irreversible. Now, writing in Science, Dorn and colleagues identify first-in-class mitofusin agonists which normalized axonal mitochondrial trafficking in sciatic nerves of a preclinical mouse CMT2A model.

MFN1 and MFN2 are GTPases found in the mitochondrial outer membrane that promote mitochondrial fusion and subcellular trafficking. Previous studies have suggested

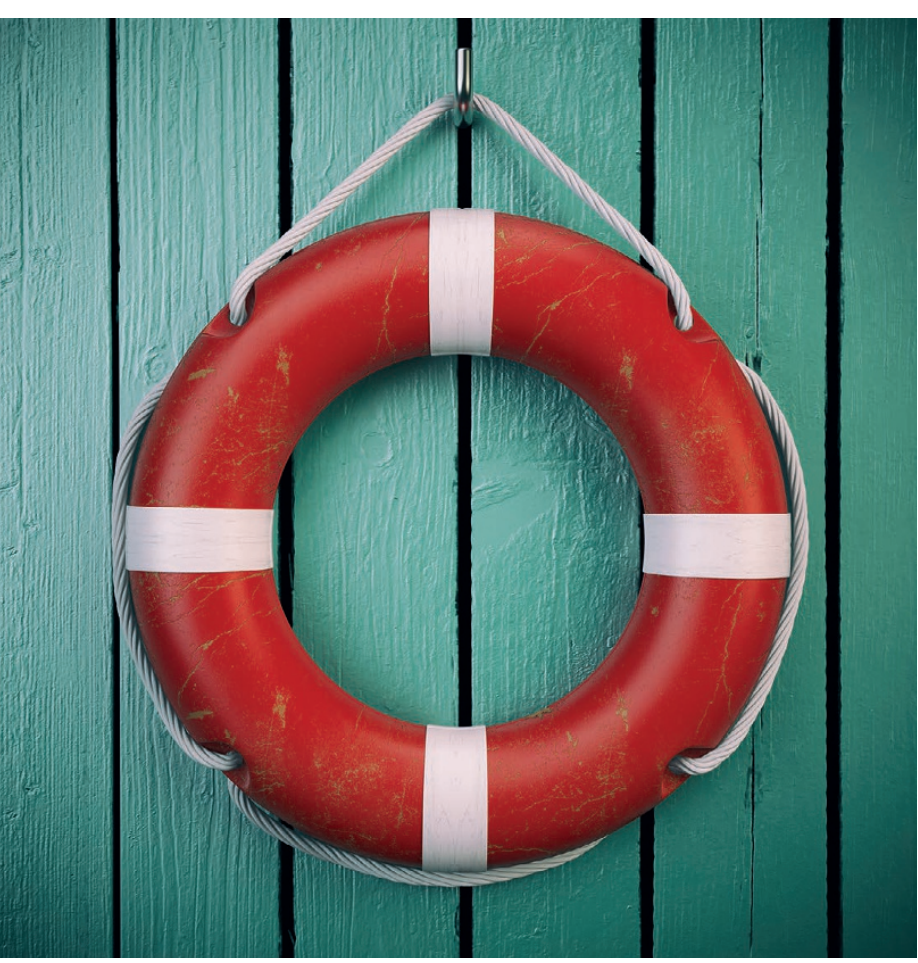

Maksym Yemelyanov/Alamy Stock Photo that MFN2 can change conformation - a closed conformation is fusion incompetent, with an open conformation favouring mitochondrial fusion.

To identify amino acids controlling these events, the authors first carried out truncation analysis of the MFN2 HR1 domain, which defined the smallest fusion-promoting minipeptide - residues 374 to 384 . Functional investigation of this minimal peptide by alanine scanning revealed that Met376, Ser378, His380 and Met381 were required for minipeptidestimulated mitochondrial fusion.

Structural modelling of human MFN2 in a closed conformation revealed a helical interaction between the HR1 and HR2 domains, which was stabilized by alignment of Met376 and His380 side chains in the HR1 domain to Leu727 and Asp725 in the HR2 domain. Further analysis revealed that this interaction was controlled by phosphorylation of adjacent MFN2 Ser378 by phosphatase and tensin homologue (PTEN)-induced putative kinase 1 .

Next, the authors assessed fusogenic activity of commercially available small-molecule candidate pharmacophores, focusing on those with structures that mimic Ser378-phosphorylated (class A) and Ser378-unphosphorylated (class B) minipeptide amino acid side chains. Lead compound A (CpdA) and lead CpdB acted synergistically to promote mitochondrial fusion, which lead the authors to create a CpdA-CpdB chimaera. The small molecule mitofusin agonists (CpdA, $\mathrm{CpdB}$ and the chimaera) competed for minipeptide binding at the MFN2 HR2 interaction site and promoted an open MFN2 conformation, resulting in potent stimulation of mitochondrial fusion in vitro.
In mouse embryonic fibroblasts, the mitofusin agonists corrected the mitochondrial dysmorphology and reversed the mitochondrial hypopolarization induced by MFN2 mutants. Mitofusin agonists also reversed mitochondrial fragmentation and hypopolarization in cultured mouse neurons expressing the CMT2A mutants MFN2-R94Q or MFN2-T105M.

The authors next tested the effects of mitofusin agonism on neuronal mitochondrial trafficking. The chimaera reversed mitochondrial clumping and restored mitochondrial motility in cultured mouse neurons expressing MFN2-T105M. Mitochondrial hypopolarization and increased autophagy and mitochondrial dysmorphology were concomitantly ameliorated.

Finally, as clinical CMT2A classically affects long nerves innervating the lower and upper limbs, the authors evaluated the effect of mitofusin activation on in vivo axonal mitochondrial trafficking in sciatic nerves of mice expressing MFN2-T105M. Application of the chimaera to MFN2-T105Mexpressing sciatic nerves restored mitochondrial motility to within normal levels.

Together, these findings highlight the potential of mitofusin agonists in the treatment of CMT2A. Such agents may also have applications in other neurological conditions associated with mitochondrial dysmotility and fragmentation, such as Alzheimer, Parkinson and Huntington diseases.

Sarah Crunkhorn

ORIGINAL ARTICLE Rocha, A. G. et al. MFN2 agonists reverse mitochondrial defects in preclinical models of Charcot-Marie-Tooth disease type 2A. Science 360, 336-341 (2018) 